\title{
Automatic Evaluation of the Degree of Facial Nerve Paralysis
}

\author{
Ting Wang, Shu Zhang, Hui Yu, Junyu Dong*, Li-An Liu
}

\begin{abstract}
Facial paralysis affects both mental and physical health of patients. Evaluation of facial paralysis in clinical practice is normally based on the static facial asymmetry at the maximal movement state and the dynamic change of facial muscle movement. However, most existing research only considers one of these two aspects when evaluating the degree of facial paralysis. This will result in an incomplete utilization of the diagnosis information leading to a low evaluation accuracy, or even misjudgment. In this paper, a novel method is presented for evaluating the degree of facial paralysis considering both static facial asymmetry and dynamic transformation factors. A quantitative approach of static facial asymmetry based on local mirror asymmetry is proposed. The method compares the differences of the corresponding local regions between both sides of the face. This makes it effective analyzing the left and right side asymmetry for abnormal faces. A quantitative evaluation of static facial asymmetry is achieved through three steps: localization of local facial regions, extraction of asymmetry features and quantification of bilateral facial asymmetry. Once the static facial asymmetry is quantified, its dynamic counterparts can be calculated using the speed of changings in different regions caused by facial muscle movement. Then we combine the static and dynamic quantification results to evaluate the degree of facial nerve paralysis. The efficiency and effectiveness of the proposed method have been tested using our facial paralysis database with 62 patients. The experiments show that our method produced encouraging performance compared with ground truth.
\end{abstract}

Keywords Facial Nerve Paralysis· Facial Asymmetry Quantification • Facial Movement $\cdot$ Facial Grading system

\author{
T. Wang $\cdot$ S. Zhang \\ Ocean University of China, Qingdao, China \\ University of Portsmouth, Portsmouth, UK \\ E-mail: qdwangting@ouc.edu.cn \\ H. Yu \\ University of Portsmouth, Portsmouth, UK \\ E-mail: hui.yu@port.ac.uk \\ J. Dong $(\square)$ \\ Ocean University of China, Qingdao, China \\ E-mail: dongjunyu@ouc.edu.cn \\ Tel: +86-532-66782300 \\ L. Liu \\ Qingdao Hiser Medical Center, Qingdao, China \\ E-mail: qdliulian@126.com
}




\section{Introduction}

The evaluation of facial nerve functionality needs the stimulation of the shrinking of facial muscles. There are two methods to stimulate the facial muscles: external electrical stimulation and active muscle shrinking. Active muscle shrinking is often obtained by asking patients to perform facial expressions following the verbal instructions. For electrical stimulation, the residual facial motion cannot be evaluated for patients with incomplete facial paralysis, and the functionality of different facial nerve branches cannot be evaluated either.

The evaluation of facial nerve functionality based on active muscle shrinking can be conducted by using subjective and objective methods [10]. The subjective methods refer to a variety of grading schemes for facial nerve functionality in clinical practice, among which the most widely used one is House-Brackmann facial nerve grading [16]. The objective method aims at the use of objective measurements to reduce errors caused by the subjective method. According to measurement ways, the objective evaluation methods can be divided into two categories, i.e. linear measurement method and image subtraction method.

Burres et al. $[4,5]$ used linear measurement for grading facial paralysis. They manually measured the key points by simply placing a ruler on the patient's face. There are also many other researchers $[20,24,27]$ who attempted to simplify the linear measurement procedure. Based on the data generated in the maximal static response test in clinical practice, BajajLuthra et al. [3] developed a method that only uses anatomic or non-anatomic motion in the paralyzed face to quantify facial motion components. Johnson et al. [19] evaluated the possibility of functional free muscle transfer for patients with chronic facial paralysis using the method proposed in [3]. All of these researches did not involve any digital technology. In contrast, methods based on image subtraction mainly use digital images to analyze changes in certain facial areas. Neely et al. [25] used a video camera to capture facial expressions and analyzed the difference between the facial expression frame and the reference image frame with the expressionless face. Meier-Gallati et al. [23] developed a scaling method for facial nerve functionality evaluation, which used variations of regional luminance produced by changing facial expressions.

In this paper, we present a novel method for automatically evaluating the degree of facial paralysis by considering both static and dynamic facial asymmetries. A quantitative method for evaluating facial symmetry is proposed, which extracts the differences between left and right half-faces with different facial expressions. This method is effective to analyze facial asymmetry for both sides of abnormal faces. It is achieved through three steps: location of local facial regions, extraction of the asymmetry features and quantification of static bilateral facial asymmetry. Then, the dynamic face asymmetry quantification can be expressed by using the change of speed in corresponding regions for each facial muscle movement. At last, we combine the static and dynamic asymmetry quantification results to evaluate the degree of facial nerve paralysis. We used a facial paralysis database with 62 patients to test the efficiency and effectiveness of the proposed method.

The rest of the paper is organized as follows. We first review related work in Section 2. Then we describe feature extraction methods on static and dynamic facial asymmetry in Section 3 and Section 4, respectively. Section 5 presents experiments and results. Finally, the paper is concluded in Section 6 with a short description of future work.

\section{Related work}

Several grading quantification methods for evaluating facial paralysis have been proposed. Liu et al. [21] used SUSAN edge detection algorithms to detect the edges of eyes and wrinkles. It used the OTSU algorithm, based on the assumption that the image to be threshold contains two classes of pixels, to obtain the mouth and nostril areas. Then they estimated the 
degree of facial paralysis by comparing the number of pixels within certain regions from two sides of face. Dong et al. [8,9] calculated the difference between the two facial sides based on key points. He et al. [13] presented an algorithm to calculate the optical flow to obtain motion features. In [14] they described the symmetry evaluation of facial movements by the resistor-average distance. He et al. $[13,14]$ only cropped the regional image sequence in the frames corresponding to its relevant movement for the paralysis analysis. In their work, they recorded the patient's facial movement information in videos. Five movements were performed in a fixed sequence with a neutral face placed in the interval of every two movements. They used the image subtraction algorithm to locate the start and end positions of each movement in the video. McGrenary [22] also used a neutral face to separate each movement, and then used the maximum displacement values and mean intensities of each region as features to grade the facial paralysis. Gebhard [11] extracted 12 parameters embedded in five sets corresponding to five movements respectively. He used these parameters to classify facial paralysis. Isono et al. [17] tracked muscle motion in expressions based on 24 markers placed on the facial surface. In the closing-eyes expression, they quantitatively analyzed the distances of muscle movement on both left and right sides respectively. Wang et al. [31] used the Pface to define the asymmetry of facial paralysis and used eigen-flow to measure the expression variations between the patient and a normal person. There are some researches [28, 30] evaluating the ability of Automated Facial Analysis (AFA), which uses many processing methods such as dense flow for detecting motions, the Lucas-Kanada algorithm for tracking facial features, high-gradient component detection for wrinkles and furrow feature extraction, and motion change detection of facial paralyzed patients. AFA enables recognition of subtle changes in facial movement. However, facial features that AFA required are manually marked in the first frame of the image sequence. There are also some systems using 3D information to grade the degree of facial paralysis. Hontanilla [15] provided 3D movement information of facial vectors to quantitatively analyze evaluation of facial movement by asking the patient to perform several face movements such as smile, mouth-puckering, eyes-closing and forehead-elevating. In [12] two facial movements i.e. eye-closing and grin are analyzed. The asymmetry evaluation was based on comparison of deformation and orientation, in both left and right sides of $3 \mathrm{D}$ expression models composed by triangular patches.

The majority of existing researches follow some requirements: (a) evaluation is under the condition of prior knowledge of facial movement patterns [1, 2, 7-9, 18], or (b) evaluation is carried on with a pre-defined or fixed sequence of different facial movement patterns recorded in the video, with a rest face between each facial movement for motion discrimination [11, 13, 14, 22]. Most methods mentioned above cannot work well for evaluating the degree of facial paralysis under unlabeled image data or under disordered sequence video data. Moreover, combination of static and dynamic quantification results to evaluate the facial paralyzed degree is hardly used in existing research. In this paper, we propose a novel method for automatically evaluating the degree of facial paralysis according to both static facial asymmetry and dynamic facial changes.

\section{Extraction of the static facial asymmetry}

Patients with either single-side or both-side facial paralysis and with different degrees were studied in the hospital of Hiser Medical Center. In total, our facial paralysis database contains 62 patients with 33 females and 29 males. The age of the subjects ranges from seven to sixtyeight years old. The equipment for data collection is shown in the Fig. 1. 


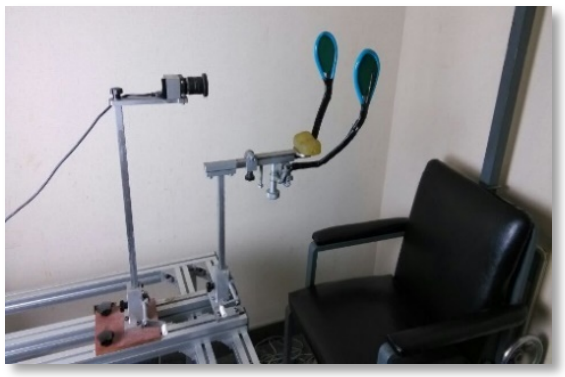

(a)

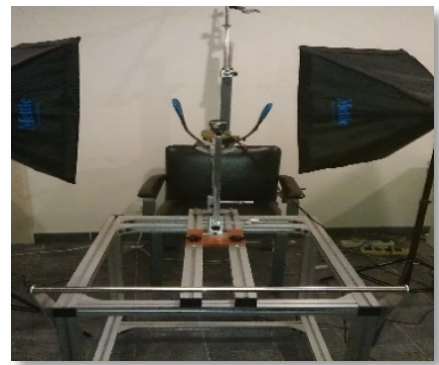

(b)

Fig. 1 Illustration of the imaging system for collecting facial images. (a) The equipment can fix the head and prevent the large variety in facial pose; (b) The surface light source, which is used to approximate nature light, can reduce the shadows on the face.

\subsection{Normalization of facial paralysis data}

The imaging conditions in the hospital are less constrained, so it gives high flexibility to doctors and patients for diagnosis and data collection. However, these flexible constraints also allow the patient to choose a more comfortable posture during facial data collection, which brings much variance to the scale and angle of face recorded in the database. In order to solve the above problems, the collected data need to be normalized through the follow steps.

Firstly, we use the ASMs algorithm, proposed by Cootes and Taylor [6], to locate the positions of pupils and the position of the apex of nose. The midline of face is defined as the line across the midpoint of two pupils and the apex nasi, as illustrated in Fig. 2.

Secondly, we rotate the image plane to set the midline of face in the vertical direction.

Thirdly, we scale the distance of pupils to 68 pixels, the length between the left pupil and the top of the image in the vertical direction is scaled to 85 pixels.

Then, all facial data are normalized in the same scale that can easily compare with different faces. Each image is cropped into a $138 \times 196$ rectangle.

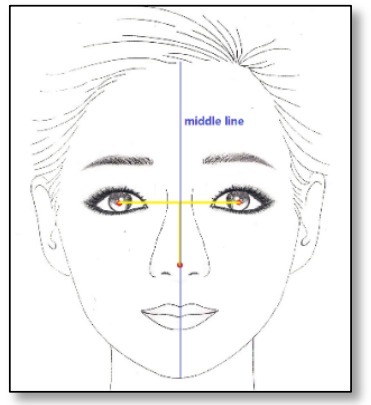

Fig. 2 The demonstration of facial image normalization.

\subsection{Localization of facial local regions}

We use the Facial Paralysis ASMs (FPASMs) to locate 68 key points on the face image of a facial paralyzed patient. The position of 68 facial key points are illustrated in Fig. 3 . The FPASMs is trained using facial paralysis images, each of which has been annotated with 68 
landmarks. Then the trained model is used to fit a new paralyzed face. The fitting result of the new patient is illustrated in Fig. 3. (This illustration is from [32]).
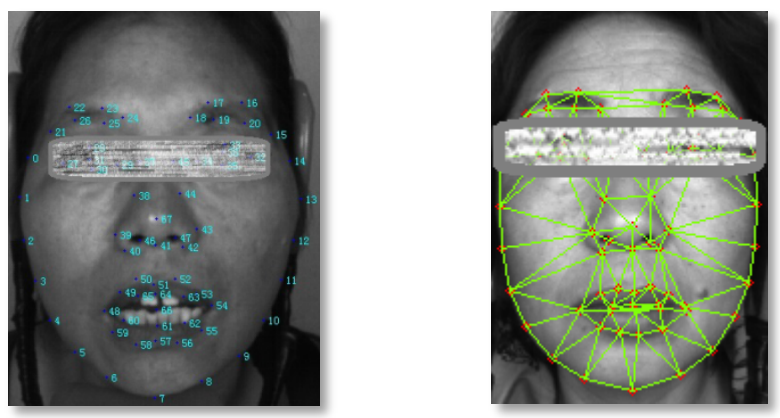

Fig. 3 Illustration of the position of 68 key points and the fitting result by using FPASMs.

There are many variations on the paralyzed face because a number of causes may result in different facial nerve paralysis. These variations lead to the fact that positions of the 68 key points cannot fit precisely on the face of facial paralyzed patients. Therefore, we extract texture features on the local facial regions. We divide the face into eight regions, as shown in Fig. 4. These eight facial regions are segmented by the follow steps. Firstly, we compute the longest width $w$ and the highest height $h$ of these eight facial rectangle regions respectively from all train data, including the Eyebrow region, Left-Eye region, Right-Eye region, UpperNose region, Low-Nose region, Left-Cheek region, Right-Cheek region and Mouth region. Since different movements can produce changes on the shapes and positions of facial organs and the regions will be also changed, we expand the width $w$ and height $h$ of each facial organ region in different scales in a similar way to previous work [32]. Secondly, we define the locations of each facial organ based on the rule that the facial organ should be in the center of the rectangle region with a dynamic displacement ( $\alpha_{n}$ along the $\mathrm{x}$-axis and $\beta_{n}$ along the y-axis). We define eight equations to obtain the coordinates of the upper-left corner of the value of each region.

The upper-left corner of the Eyebrow rectangle region is point B1, and the coordinates of point $\mathrm{B} 1\left(x_{\mathrm{P}_{\mathrm{B} 1}}, y_{\mathrm{P}_{\mathrm{B} 1}}\right)$ can be located based on Eq. (1).

$$
x_{\mathrm{P}_{\mathrm{B} 1}}=x_{\mathrm{P}_{21}}-\alpha_{1}, \quad y_{\mathrm{P}_{\mathrm{B} 1}}=y_{\mathrm{P}_{22}}-\beta_{1}
$$

The upper-left corner of the Left-Eye rectangle region is point $\mathrm{B} 2$ and that of the RightEye region is point $\mathrm{B} 3$, and the coordinates of $\mathrm{B} 2\left(x_{\mathrm{P}_{\mathrm{B} 2}}, y_{\mathrm{P}_{\mathrm{B} 2}}\right)$ and $\mathrm{B} 3\left(x_{\mathrm{P}_{\mathrm{B} 3}}, y_{\mathrm{P}_{\mathrm{B} 3}}\right)$ can be located using Eq. (2) and Eq. (3) respectively.

$$
\begin{array}{ll}
x_{\mathrm{P}_{\mathrm{B} 2}}=x_{\mathrm{P}_{27}}-\alpha_{2}, & y_{\mathrm{P}_{\mathrm{B} 2}}=y_{\mathrm{P}_{28}}-\beta_{2} \\
x_{\mathrm{P}_{\mathrm{B} 3}}=x_{\mathrm{P}_{32}}+\alpha_{3}, & y_{\mathrm{P}_{\mathrm{B} 3}}=y_{\mathrm{P}_{33}}-\beta_{3}
\end{array}
$$

The upper-left corner of the Upper-Nose rectangle region is point B4 and that of the Lower-Nose region is point $\mathrm{B} 5$, and the coordinates of $\mathrm{B} 4\left(x_{\mathrm{P}_{\mathrm{B}}}, y_{\mathrm{P}_{\mathrm{B} 4}}\right)$ and $\mathrm{B} 5\left(x_{\mathrm{P}_{\mathrm{B} 5}}, y_{\mathrm{P}_{\mathrm{B} 5}}\right)$ can be located based on Eq. (4) and Eq. (5) respectively.

$$
\begin{array}{ll}
x_{\mathrm{P}_{\mathrm{B} 4}}=x_{\mathrm{P}_{37}}-\alpha_{4}, & y_{\mathrm{P}_{\mathrm{B} 4}}=y_{\mathrm{P}_{29}}-\beta_{4} \\
x_{\mathrm{P}_{\mathrm{B} 5}}=x_{\mathrm{P}_{\mathrm{B} 4}}-\alpha_{5}, & y_{\mathrm{P}_{\mathrm{B} 5}}=y_{\mathrm{P}_{\mathrm{B} 4}}+\beta_{5}
\end{array}
$$

Similar to the region of Eye, the upper-left corner of the Left-Cheek rectangle region is 
point $\mathrm{B} 6$ and that of the Right-Cheek region is point $\mathrm{B} 7$, and the coordinates of $\mathrm{B} 6\left(x_{\mathrm{P}_{\mathrm{B} 6}}\right.$, $\left.y_{\mathrm{P}_{\mathrm{B} 6}}\right)$ and $\mathrm{B} 7$ ( $\left.x_{\mathrm{P}_{\mathrm{B} 7}}, y_{\mathrm{P}_{\mathrm{B} 7}}\right)$ can be located based on Eq. (6) and Eq. (7) respectively.

$$
\begin{array}{ll}
x_{\mathrm{P}_{\mathrm{B}}}=x_{\mathrm{P}_{2}}-\alpha_{6}, & y_{\mathrm{P}_{\mathrm{B} 6}}=y_{\mathrm{P}_{1}}-\beta_{6} \\
x_{\mathrm{P}_{\mathrm{B} 7}}=x_{\mathrm{P}_{12}}+\alpha_{7}, & y_{\mathrm{P}_{\mathrm{B} 7}}=y_{\mathrm{P}_{13}}-\beta_{7}
\end{array}
$$

The upper-left corner of the Mouth rectangle region is point $\mathrm{B} 8$, and the coordinates of $\mathrm{B} 8$ $\left(x_{\mathrm{P}_{\mathrm{B} 8}}, y_{\mathrm{P}_{\mathrm{B} 8}}\right)$ can be located based on Eq. (8).

$$
x_{\mathrm{P}_{\mathrm{B} 8}}=x_{\mathrm{P}_{48}}-\alpha_{8}, \quad y_{\mathrm{P}_{\mathrm{B} 8}}=y_{\mathrm{P}_{50}}-\beta_{8}
$$

The correlative key points and parameters for defining each region is illustrated in Fig. 5. The detail of locating the eight regions can be found in [32].

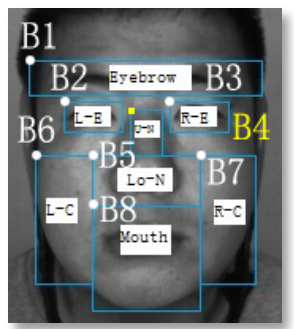

Fig. 4 Diagram of facial regions. Note: Eyebrow: Eyebrow region, E: Eye region, N: Nose region, C: Cheek region, Mouth: mouth region, L: Left, R: Right, U: Upper, Lo: Lower.

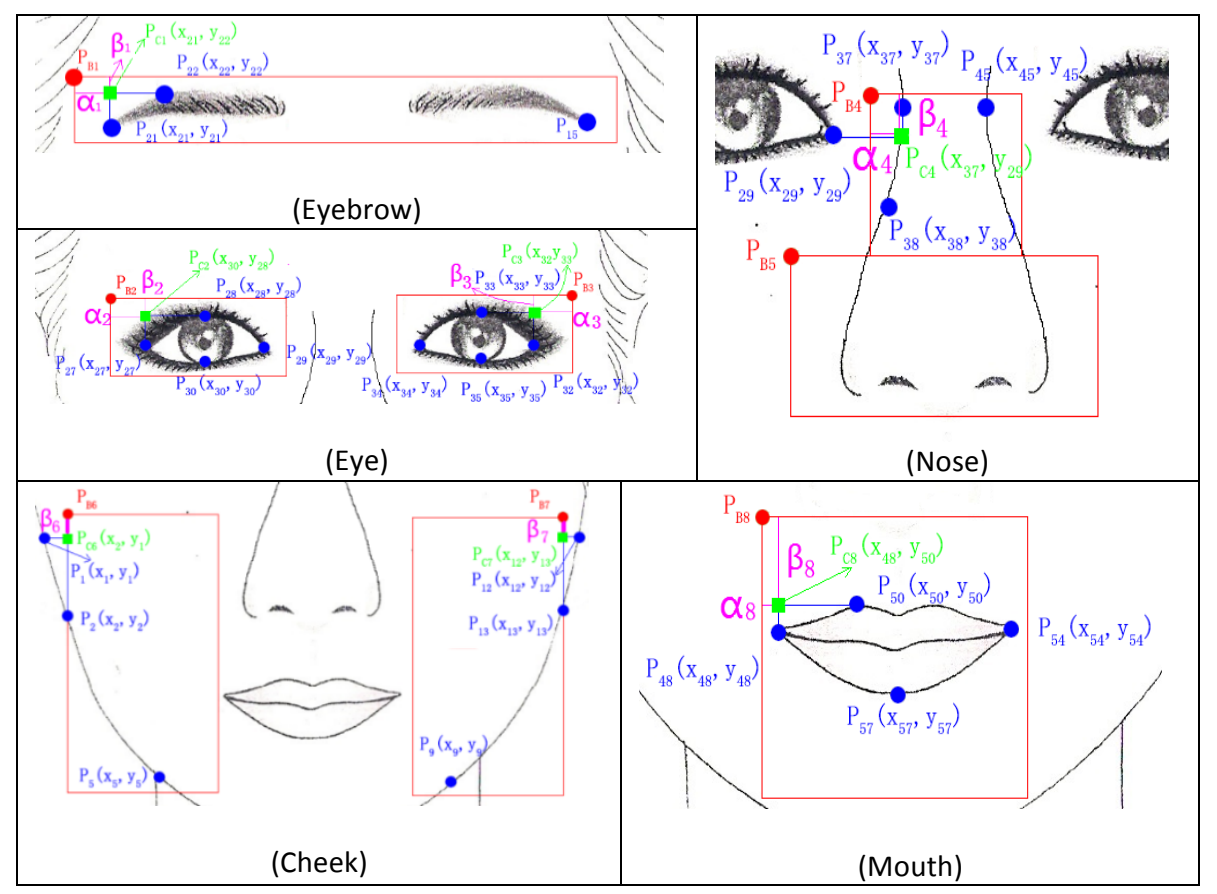

Fig. 5 Illustration of the points and parameters which are used to locate the eight rectangle regions. 
3.3 Extraction of the asymmetry features

The House-Brackmann score [16] is used to grade the degree of nerve damage in a facial nerve palsy. The measurement is determined by measuring the upwards movement of the mid-portion of the top of the eyebrow, and the outwards movement of the angle of the mouth. In our facial paralyzed database, these patients performed 5 facial movements (raiseeyebrows, close-eyes, screw-up-nose, plump-cheeks and open-mouth). In different facial movements, each facial organ draws different degrees of concern. For example, the doctor may focus on the change of the eyebrows region and the eye region when the patient preforms raise-eyebrows expression. In this paper, we extract facial asymmetry features from a combination of regions according to each facial movement. The rule of combination of organ regions to form fused regions for the five facial movements $\operatorname{Reg}_{\text {movement }}$, is defined as follow:

1) The region for raise-eyebrows ( $\left.R e g_{\text {raise-eyebrows }}\right)$ is composed of regions of eye, and eyebrow;

2) The region for close-eyes $\left(\operatorname{Reg}_{\text {close-eyes }}\right)$ consists of regions of eyebrow, eye, and upper nose;

$3)$ The region for screw-up-nose $\left(\right.$ Reg $\left._{\text {screw-up-nose }}\right)$ consists of regions of eye, upper nose, and lower nose;

4) The region for plump-cheek ( Reg $\left._{\text {plump-cheek }}\right)$ consists of regions of lower nose, and cheek;

5) The region for open-mouth $\left(\operatorname{Reg}_{\text {open-mouth }}\right)$ consists of regions of lower nose, cheek, and mouth.

Based on the previous work on recognition of the facial movement patterns [32], this method can automatically obtain Reg $_{\text {movement }}$ according to different facial movements. We subtract the left regions from the corresponding regions in the right side to generate the difference region $D_{O}, O \in\{$ Eyebrow, Eye, Upper-Nose, Lower-Nose, Cheek, Mouth $\}$. We define the difference region $D_{O}$ is

$$
\mathrm{D}_{O}=\left|L_{O}-R_{O}\right|
$$

where $L_{O}$ refers to the region of the facial organ in the left side of a face. $R_{O}$ is the reflected region of the facial organ in the right half face, which can be obtained according to Fig. 6.

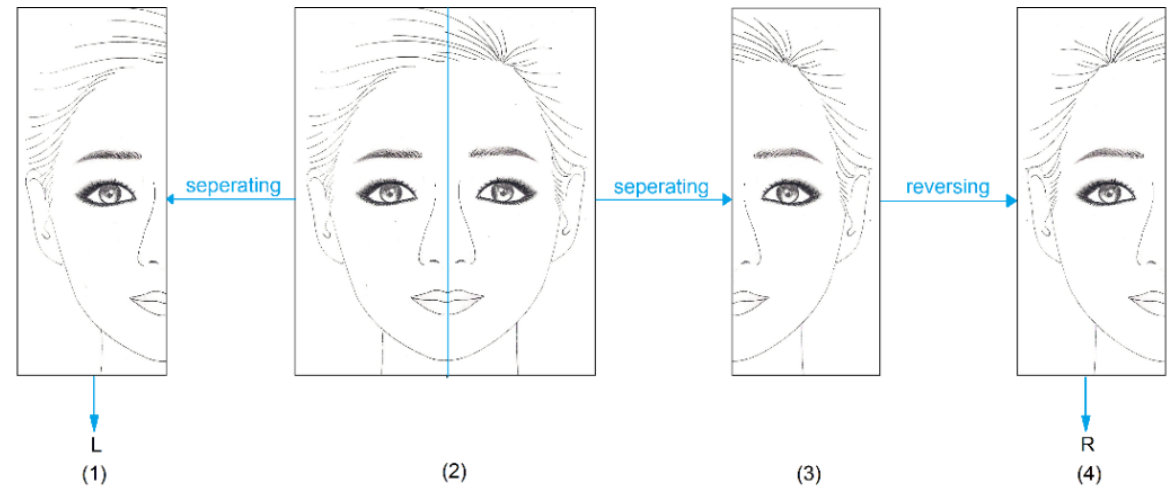

Fig.6 Illustration of "L" and "R". (1) and (3) are the separated left face and right face from (2) respectively; the (4) is the reflection of (3).

We extract the LBP features from a combination of regions according to each facial movement, and concatenate these LBP feature histograms to represent facial asymmetry of the left and right side of a face. The LBP feature was originally proposed for texture analysis by Ojala et al. [26]. The extraction of LBP feature of five facial movements $L B P_{\text {movement }}$ from $\operatorname{Reg}_{\text {movement }}$ are 


$$
\begin{aligned}
& L B P_{\text {raise-eyebrows }}=\text { Concatenate }\left(L B P_{\left|D_{\text {eyebrow }}\right|}, L B P_{\left|D_{\text {eve }}\right|}\right) \\
& L B P_{\text {close-eyes }}=\text { Concatenate }\left(L B P_{\left|D_{\text {everbow }}\right|}, L B P_{\left|D_{\text {eye }}\right|}, L B P_{\left|D_{\text {upper-nose }}\right|}\right) \\
& L B P_{\text {screw-up-nose }}=\text { Concatenate }\left(L B P_{\left|D_{\text {eye }}\right|}, L B P_{\left|D_{\text {upper-nose }}\right|}, L B P_{\left|D_{\text {lower-nose }}\right|}\right) \\
& L B P_{\text {plump-cheeks }}=\text { Concatenate }\left(L B P_{\left|D_{\text {lower-nose }}\right|}, L B P_{\left|D_{\text {cheek }}\right|}\right) \\
& L B P_{\text {open-mouth }}=\text { Concatenate }\left(L B P_{\mid D_{\text {lower-nose }},}, L B P_{\left|D_{\text {cheek }}\right|}, L B P_{\left|D_{\text {mouth }}\right|}\right)
\end{aligned}
$$

The operator $L B P_{8,2}^{u 2}$ is selected to extract features of these regions in this paper.

\section{Extraction of movement asymmetry}

\subsection{Recognition of key frames}

The facial paralysis data is stored in image sequences. On average, each full facial movement is captured under the fps of 15 . In each image sequence, a patient performs a particular facial movement starting from a neutral expression, and then gradually shifts to the maximum status of the facial movement, and then return to a neutral expression in the end. Our method automatically recognizes the key movement in an image sequence under the following condition: the neutral state or the maximal state of an expression should last more than 4 continuous frames. This is equivalent to 0.27 second, which is very convenient to be conducted in practice.

\subsection{Extraction of dynamic change feature}

In each image sequence, we define the last image of the neutral expression in a continuous collection of images to be the referenced neutral image $\left(I_{\text {neutral }}\right)$, and the first image of the maximal movement state in a continuous collection of images to be the referenced maximum image $\left(I_{\max }\right)$. Then we locate the frame positions of the referenced neutral image $\left(\right.$ Pos $\left._{I_{\text {neutral }}}\right)$ and the referenced maximum image $\left(\operatorname{Pos}_{I_{\max }}\right)$ in the image sequence. $P o S_{I_{\max }}$ is of the same value of $\operatorname{Pos}_{I_{\text {neutral }}}$ until maximal movement state is found in the image sequence. We extract the dynamic change features by calculating the number of frames $\left(N_{\text {change }}\right)$ from the referenced neutral image to the referenced maximum image in the image sequence, as in Eq. (15). Note that, the value of $N_{\text {change }}$ is zero when the patient is fully facial paralyzed.

$$
N_{\text {change }}=\operatorname{Pos}_{I_{\max }}-\operatorname{Pos}_{I_{n}}
$$

We extract the dynamic change features $N_{\text {change }}$ from the left side and right side of the face respectively, and quantify the dynamic asymmetry $\left(Q_{\text {dynamic }}\right)$ as a ratio of the difference between the left side and right side of the face. Note that, the value of $Q_{\text {dynamic }}$ is one when the degree of facial paralysis is grade VI, while $Q_{\text {dynamic }}$ is zero when the degree of facial paralysis is grade I. The zero value for $\max \left(L_{N_{\text {change }}}, R_{N_{\text {change }}}\right)$ means that patient has not perform any facial movement with noticeable visual changes, which imply that this patient has facial paralysis in the grade of VI who cannot move his/her facial muscle.

$$
\begin{cases}Q_{\text {dynamic }}=1.0 & , \max \left(L_{N_{\text {change }}}, R_{N_{\text {change }}}\right)=0 \\ Q_{\text {dynamic }}=\frac{\left|L_{N_{\text {change }}}-R_{N_{\text {change }} \mid}\right|}{\max \left(L_{N_{\text {change }}}, R_{N_{\text {change }}}\right)}, \max \left(L_{N_{\text {change }}}, R_{N_{\text {change }}}\right) \neq 0\end{cases}
$$




\section{Quantification of the facial asymmetry}

Once the facial asymmetry is obtained, we quantify the facial asymmetry to evaluate the degree of facial paralysis.

\subsection{Quantification of facial bilateral difference}

Since the doctors' grading standard is based on a percentage scale, we quantify the static facial asymmetry $\left(Q_{\text {static }}\right)$ according to the transformation relationship between $Q_{\text {static }}$ and Doctors' grading standard as in Tab.1. We use the extracted $L B P_{\text {movemet }}$ and $Q_{\text {static }}$ to train an SVM classifier, which aims at establishing the relationship between $L B P_{\text {movemet }}$ and $Q_{\text {static }}$. Then we use the trained SVM to obtain the value of $Q_{\text {static }}$, which corresponds to the extracted $L B P_{\text {movemet }}$.

Table 1 The transformation relationship between the quantified static asymmetry and Doctors' grading standard

\begin{tabular}{lccccccc}
$\begin{array}{l}\text { The quantified static } \\
\text { asymmetry }\left(Q_{\text {static }}\right)\end{array}$ & 5 & 13 & 20 & 28 & 33 & 40 & 48 \\
\cline { 1 - 8 } Doctors' grading standard $(x)$ & {$[0,10]$} & $(10,15]$ & $(15,25]$ & $(25,30]$ & $(30,35]$ & $(35,45]$ & $(45,50]$ \\
\cline { 1 - 5 } $\begin{array}{l}\text { The quantified static } \\
\text { asymmetry }\left(Q_{\text {static }}\right)\end{array}$ & 53 & 60 & 68 & 73 & 80 & 88 & 95 \\
\cline { 1 - 6 } Doctors' grading standard $(x)$ & $(50,55]$ & $(55,65]$ & $(65,70]$ & $(70,75]$ & $(75,85]$ & $(85,90]$ & $(90,100]$ \\
\hline
\end{tabular}

5.2 Quantification of dynamic change difference

We quantify the dynamic facial asymmetry based on the transformation relationship between $Q_{\text {dynamic, }}$, defined in Eq. (16), and Doctors' grading standard as in Tab.2, calculated based on the statistics of the data. The $Q_{d y n a m i c}$ and $x$ are a linear relationship in each segment.

Table 2 The transformation relation between the quantified dynamic asymmetry and Doctors' grading standard

\begin{tabular}{lcccccc}
\hline $\begin{array}{l}\text { The quantified dynamic } \\
\text { asymmetry }\left(Q_{\text {dynamic }}\right)\end{array}$ & {$[0.0,0.1]$} & $(0.1,0.3]$ & $(0.3,0.5]$ & $(0.5,0.7]$ & $(0.7,0.9]$ & $(0.9,1.0]$ \\
\cline { 1 - 4 } $\begin{array}{l}\text { Doctors' grading } \\
\text { standard }(x)\end{array}$ & {$[0,10]$} & $(10,30]$ & $(30,50]$ & $(50,70]$ & $(70,90]$ & $(90,100]$ \\
\hline
\end{tabular}

5.3 Quantification of holistic facial asymmetry

We evaluate the degree of facial paralysis by combining the quantification of dynamic facial asymmetry $\left(Q_{\text {dymatic }}\right)$ and the quantification of static facial asymmetry $\left(Q_{\text {static }}\right)$. The quantification of holistic facial paralysis asymmetry $\left(Q_{\text {holistic }}\right)$ is define in the Eq. (17).

$$
Q_{\text {holistic }}=\lambda \cdot Q_{\text {static }}+(1-\lambda) \cdot \operatorname{Transfrom}\left(Q_{\text {dynamic }}\right)
$$

where the function of Transform $\left(Q_{\text {dymatic }}\right)$ transforms the grading score from $Q_{\text {dymatic }}$ to Doctor' grading standard according to Tab. 2 . The parameter $\lambda$ refers to the ratio between the static component and the dynamic component of the quantification. Its value can be assigned according to clinical experience from the doctors' evaluations. Typically, $\lambda$ should be larger than $1 / 2$ to allow the static component to contribute more. In our study, we have found that the choice of $3 / 4$ for $\lambda$ would result in a better performance compared to the ground truth.

\section{Experiments}

We ask 10 professional doctors to grade on 62 facial paralysis patients with different grades of paralysis according to the House-Brackmann (H-B) grading system. The doctors' evaluation process is the same as the method by Wang et al. [33]. These patients perform 5 
facial movements (raise eyebrows, close eyes, screw-up nose, plump cheeks and open mouth). These movements are recorded both in achromatic images and chromatic videos. Each doctor views all these samples in a random order, and different visual data types of the same patient are shown in an interval of ten days. In our facial paralyzed database, the grade scale of facial paralysis is from I to VI. There are more than 8 patients in every grade of each facial movement in the database. To have a uniform grade representation, we ask the doctors to use a percentage grading system. The corresponding relationship between doctors' grading standards and the House-Brackmann (H-B) grading system is illustrated in the Tab. 3. The average values of 10 doctors' scores on the achromatic videos are used as the ground truth.

Support Vector Machine (SVM) has been successfully used facial expression classification [29]. In this paper, we use the SVM to classify the degrees of facial paralysis in each facial movement. The leave-one-out cross-validation strategy is used for estimating the performance of evaluation accuracy.

Table 3 The corresponding relation between doctors' grading standard and House-Brackmann (H-B) grading system.

\begin{tabular}{cc}
\hline Doctors' grading standard $(x)$ & House-Brackmann (H-B) grading system \\
\hline $0 \leq x \leq 10$ & I \\
$10<x \leq 30$ & II \\
$30<x \leq 50$ & III \\
$50<x \leq 70$ & IV \\
$70<x \leq 90$ & V \\
$90<x \leq 100$ & VI \\
\hline
\end{tabular}

We use SVM with the RBF kernel function to quantify the static facial asymmetry on the image with the maximal state in five facial movements respectively. The results are shown in Tab. 4 .

Table 4 The fitting results of static asymmetry feature with comparison to the ground truth

\begin{tabular}{cccccc}
\hline Facial Movement & $\begin{array}{c}\text { Raise } \\
\text { Eyebrows }\end{array}$ & Close Eyes & $\begin{array}{c}\text { Crew-up } \\
\text { Nose }\end{array}$ & Plump Cheeks & Open Mouth \\
\hline $\begin{array}{c}\text { RBF (recognition } \\
\text { rate \%) }\end{array}$ & $89.17 \%$ & $92.35 \%$ & $90.04 \%$ & $86.71 \%$ & $91.36 \%$ \\
\hline
\end{tabular}

After analyzing the fitting errors, we found that the misclassification usually happen in the value around the threshold. For example, the value of doctors' grading is 31 (the corresponding evaluation is III in the H-B), but the predicted value based on SVM is 28 (the corresponding evaluation is II in the H-B). This will lead to error quantification. We also found that evaluation of the degree of facial paralysis solely based on the static facial asymmetry is not good enough. In contrast, a proposed method based on combination of static quantification and dynamic quantification can obtain the highest recognition rate of $97.56 \%$ when the value $\lambda$ is 0.75 . The results are shown in the Tab.5.

Table 5 The fitting results of combination of static and dynamic asymmetry feature to the ground truth

\begin{tabular}{cccccc}
\hline Facial Movement & $\begin{array}{c}\text { Raise } \\
\text { Eyebrows }\end{array}$ & $\begin{array}{c}\text { Close } \\
\text { Eyes }\end{array}$ & $\begin{array}{c}\text { Crew-up } \\
\text { Nose }\end{array}$ & $\begin{array}{c}\text { Plump } \\
\text { Cheeks }\end{array}$ & $\begin{array}{c}\text { Open } \\
\text { Mouth }\end{array}$ \\
\hline $\begin{array}{c}\text { Evaluation of Facial Paralyzed } \\
\text { Degree (recognition rate \%) }\end{array}$ & $92.55 \%$ & $97.56 \%$ & $93.36 \%$ & $91.36 \%$ & $95.40 \%$ \\
\hline
\end{tabular}


In this paper, a new method is introduced for automatically evaluating the degree of facially paralyzed patients. In the proposed method, both of the static facial asymmetry and the dynamic asymmetry are combined together to quantify the holistic facial asymmetry based on five facial movements. First, we extract the facial asymmetry feature in images with the maximal state of movement and image sequences with full movement respectively. Then, we quantify the static asymmetry using SVM and the dynamic asymmetry using the rate of change in the left side and the right side of the face. Finally, we evaluate the degree of facial paralysis by combining the quantified results of both static and dynamic values. Experiments show that the proposed method has encouraging performance when tested using our facial paralysis database.

In future, we will conduct further research on the method of accurately locating the key points on the facial paralyzed face, and testing different distance metrics in the current method. New technologies such as RGB-D sensors can also be combined to improve the accuracy of facial palsy analysis.

\section{Acknowledgments}

This work is supported by the National Natural Science Foundation of China (No. 61271405), Ph.D. Program Foundation of Ministry of Education of China (20120132110018), China Postdoctoral Science Foundation (No. 2014M551962) and Fundamental Research Funds for the Central Universities (No. 201413020).

\section{References}

1. Anguraj K, Padma S (2012) Analysis of Facial Paralysis Disease using Image Processing Technique. International Journal of Computer Applications 54(11):1-4

2. Anguraj K, Kandiban R, Jayakumar KS (2012) Facial paralysis diseases level detection using CEM algorithm for clinical applications. Eur J Sci Res 77:543-548

3. Bajaj-Luthra A, Mueller T., Johnson PC (1997) Quantitative analysis of facial motion components: anatomic and nonanatomic motion in normal persons and in patients with complete facial paralysis. Plastic and reconstructive surgery 99(6):1894-1902

4. Burres SA (1985) Facial biomechanics: the standards of normal. The Laryngoscope 95(6):708-714

5. Burres SA (1986) Objective grading of facial paralysis. The Annals of otology, rhinology, \& laryngology 95(3):238-241

6. Cootes TF, Taylor CJ, Cooper DH, Graham J (1995) Active shape models-their training and application. Computer vision and image understanding 61(1):38-59

7. Delannoy JR, Ward TE (2010) A preliminary investigation into the use of machine vision techniques for automating facial paralysis rehabilitation therapy. Proceeding of IET Irish Signals and Systems Conference, pp 228-232.

8. Dong J, Lin Y, Liu L (2008) An Approach to Evaluation of Degree of Facial Paralysis based on Image Processing and Pattern Recognition. Journal of Information Systems 5(2):639-646

9. Dong J, Ma L, Li Q, Wang S, Liu L, Lin Y, Jian M (2008) An approach for quantitative evaluation of the degree of facial paralysis based on salient point detection. Intelligent Information Technology Application Workshops, pp 483-486

10. Dulguerov P, Marchal F, Wang D, Gysin C (1999) Review of objective topographic facial nerve evaluation methods. Otology \& Neurotology 20(5):672-678

11. Gebhard A, Paulus D (2000) Diagnosis support of patients with facial paresis. Bernd Girod G, and Niemann H. Principles of 3D Image Analysis and Synthesis. London: Kluwer Academic Publishers, pp 120

12. Gunaratne P, Sato Y (2003) Objective evaluation of facial paralysis by asymmetry in expressions. Medical Image Computing and Computer-Assisted Intervention-MICCAI 2003. Springer Berlin Heidelberg, pp 1-8

13. He S, Soraghan JJ, O'Reilly BF (2007) Biomedical image sequence analysis with application to automatic quantitative assessment of facial paralysis. Journal on Image and Video Processing, 2007(4):81282-181282-11

14. He S, Soraghan JJ, O'Reilly BF, Xing D (2009) Quantitative analysis of facial paralysis using local binary patterns in biomedical videos. IEEE Transactions on Biomedical Engineering 56(7):1864-1870 
15. Hontanilla B, Aubá C (2008) Automatic three-dimensional quantitative analysis for evaluation of facial movement. Journal of Plastic, Reconstructive \& Aesthetic Surgery 61(1):18-30

16. House JW, Brackmann DE (1985) Facial nerve grading system. Otolaryngology--head and neck surgery: official journal of American Academy of Otolaryngology-Head and Neck Surgery 93(2):146

17. Isono M, Murata K, Tanaka H, Kawamoto M, Azuma H (1996) An objective evaluation method for facial mimic motion. Otolaryngology--Head and Neck Surgery 114(1):27-31

18. Isono M, Murata K, Tanaka H, Minoyama M, Azuma H (1994) [Computerized analysis of facial motions-objective evaluation of facial palsy]. Nihon Jibiinkoka Gakkai kaiho 97(3):393-400

19. Johnson PJ, Bajaj-Luthra A, Llull R, Johnson PC (1997) Quantitative facial motion analysis after functional free muscle reanimation procedures. Plastic and reconstructive surgery 100(7):1710-1719

20. Johnson PC, Brown H, Kuzon WM, Balliet R, Garrison JL, Campbell J (1994) Simultaneous quantitation of facial movements: the maximal static response assay of facial nerve function. Annals of plastic surgery $32(2): 171-179$

21. Liu L, Cheng G, Dong J, Wang S, Qu H (2010) Evaluation of Facial Paralysis Degree Based on Regions. WKDD'10. Third International Conference on Knowledge Discovery and Data Mining, pp 514-517

22. McGrenary S, O'Reilly BF, Soraghan JJ (2005) Objective grading of facial paralysis using artificial intelligence analysis of video data. Proceedings. 18th IEEE Symposium on Computer-Based Medical Systems, pp 587-592

23. Meier-Gallati V, Scriba H, Fisch U (1998) Objective scaling of facial nerve function based on area analysis (OSCAR). Otolaryngology--Head and Neck Surgery 118(4):545-550

24. Murty GE, Diver JP, Kelly PJ, O'Donoghue GM, Bradley PJ (1994) The Nottingham System: objective assessment of facial nerve function in the clinic. Otolaryngology--Head and Neck Surgery 110(2):156-161

25. Neely JG, Cheung JY, Wood M, Byers J, Rogerson A (1992) Computerized quantitative dynamic analysis of facial motion in the paralyzed and synkinetic face. Otology \& Neurotology 13(2):97-107

26. Ojala T, Pietikainen M, Maenpaa T (2002) Multiresolution gray-scale and rotation invariant texture classification with local binary patterns. IEEE Transactions on Pattern Analysis and Machine Intelligence 24(7):971-987

27. Peckitt NS, Walker RV, Barker GR (1992) The facial nerve function coefficient: analysis of 100 normal subjects. Journal of oral and maxillofacial surgery 50(4):338-339

28. Rogers CR, Schmidt KL, VanSwearingen JM, Cohn JF, Wachtman G. S, Manders E. K, Deleyiannis F. W. B (2007) Automated facial image analysis: detecting improvement in abnormal facial movement after treatment with botulinum toxin A. Annals of plastic surgery 58(1):39-47

29. Shan C, Gong S, McOwan P. W (2009) Facial expression recognition based on local binary patterns: A comprehensive study. Image and Vision Computing 27(6):803-816

30. Wachtman G, Cohn J, Swearingen JV, Manders EK (2001) Automatic tracking of facial features in patients with facial neuromuscular dysfunction. Plastic and reconstructive surgery 107(5):1124-1133.

31. Wang S, Li H, Qi F, Zhao Y (2004) Objective facial paralysis grading based on Pface and eigenflow. Medical and Biological Engineering and Computing 42(5):598-603

32. Wang T, Dong J, Sun X, Zhang S, Wang S (2014) Automatic recognition of facial movement for paralyzed face. Bio-medical materials and engineering 24(6):2751-2760

33. Wang T, Qi L, Dong J, Zhang S, Wang S (2014) Facial paralysis grading: are achromatic images sufficient? Perception 43:19 